Supporting Information

\title{
Ultrafast Zinc-Ion Diffusion Ability Observed in 6.0- Nanometer Spinel Nanodots
}

Le Jiang ${ }^{\dagger}$, Zeyi $\mathrm{Wu}^{\dagger}$, Yanan Wang ${ }^{\dagger}$, Wenchao Tian ${ }^{\dagger}$, Zhiying $\mathrm{Yi}^{\dagger}$, Cailing Cai ${ }^{\dagger}$, Yingchang Jiang ${ }^{\dagger}$, Linfeng $\mathrm{Hu}^{\dagger^{*}}$

*Corresponding author: E-mail: linfenghu@fudan.edu.cn

Department of Materials Science, Fudan University, Shanghai 200433, P. R. China 


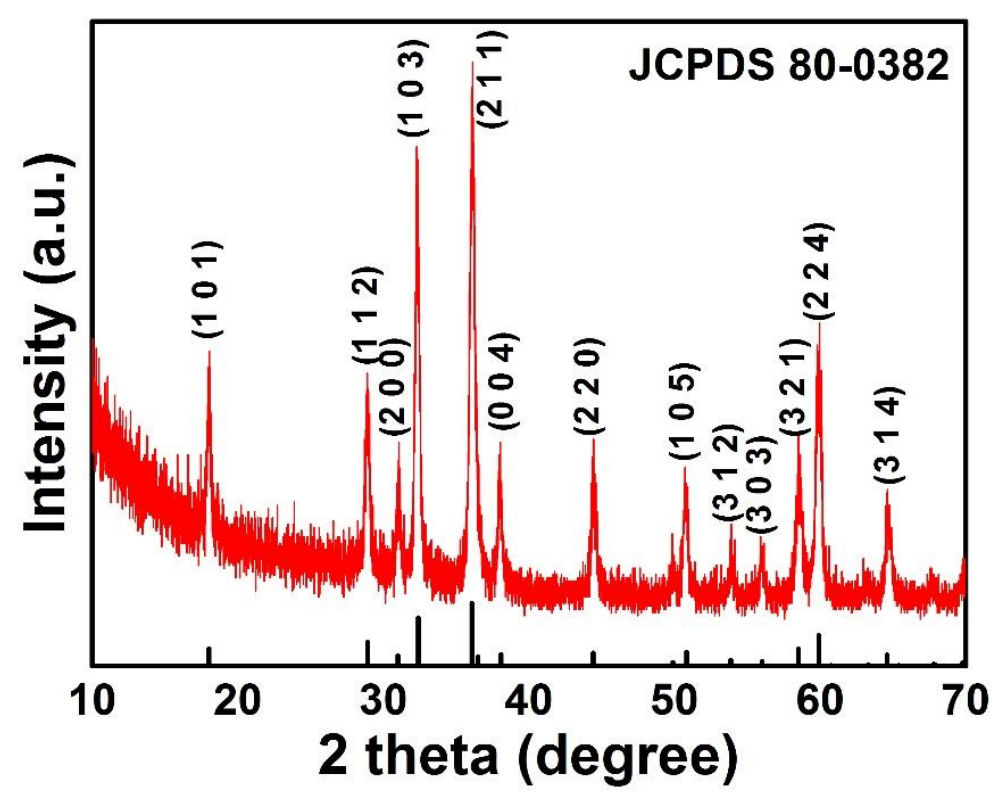

Figure S1. Typical XRD pattern of $\mathrm{Mn}_{3} \mathrm{O}_{4}$ nanostructures synthesized in $\mathrm{EtOH} / \mathrm{H}_{2} \mathrm{O}$ mixture $(v: v=1: 1)$. The diffraction peaks in Figure $\mathrm{S} 1$ are obviously shaper than those prepared in pure EtOH (Figure 1b), demonstrating larger particle diameter as the half width of diffraction peaks decrease. 

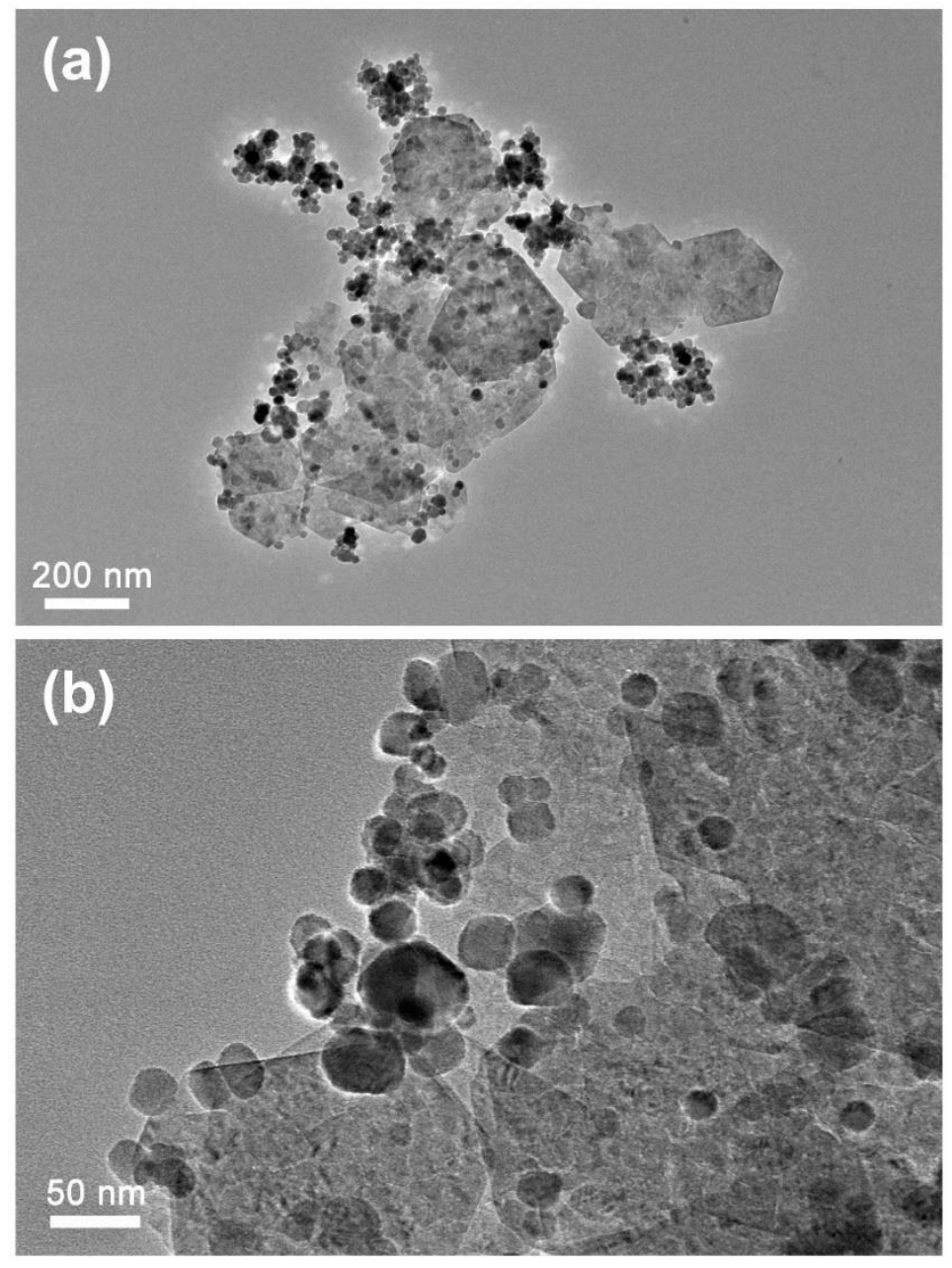

Figure S2. TEM image of $\mathrm{Mn}_{3} \mathrm{O}_{4}$ nanostructures synthesized in $\mathrm{EtOH} / \mathrm{H}_{2} \mathrm{O}$ mixture $(v: v$ $=1: 1)$, indicating an irregular morphology consisting of nanodots $(20 \sim 50 \mathrm{~nm})$ and hexagonal nanoplates. 


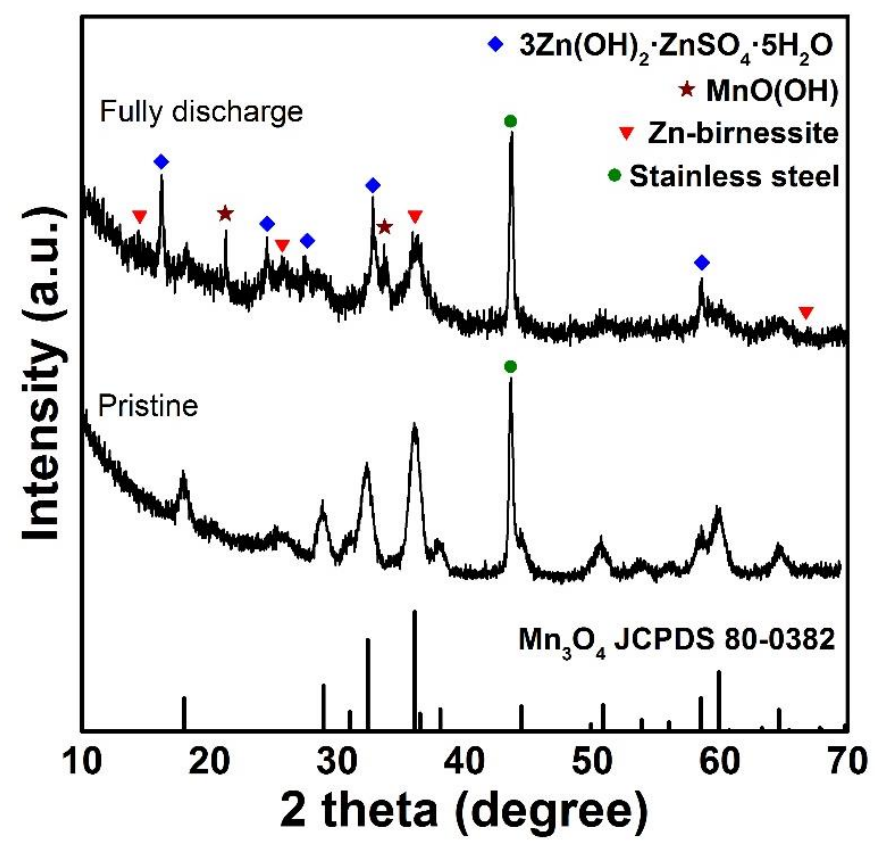

Figure S3. Ex situ XRD of $\mathrm{Mn}_{3} \mathrm{O}_{4}$ nanodots cathode before and after 100 cycles at $0.5 \mathrm{~A}$ $\mathrm{g}^{-1}$. The XRD pattern of the pristine cathode is well consistent with tetragonal $\mathrm{Mn}_{3} \mathrm{O}_{4}$ (JCPDS Card No. 80-0382). After 100 cycles at $0.5 \mathrm{~A} \mathrm{~g}^{-1}$, the peak intensity of $\mathrm{Mn}_{3} \mathrm{O}_{4}$ becomes weaker and some new diffraction peaks appear, which can be well indexed to $\mathrm{Zn}$-birnessite, $\mathrm{MnO}(\mathrm{OH})$ and $3 \mathrm{Zn}(\mathrm{OH})_{2} \cdot \mathrm{ZnSO}_{4} \cdot 5 \mathrm{H}_{2} \mathrm{O}$, respectively. According to the previous reports ${ }^{1,2}$, the consumption of $\mathrm{H}^{+}$in the electrolyte results in the formation of $\mathrm{MnO}(\mathrm{OH})$ and $3 \mathrm{Zn}(\mathrm{OH})_{2} \cdot \mathrm{ZnSO}_{4} \cdot 5 \mathrm{H}_{2} \mathrm{O}$, which provide the evidence on $\mathrm{H}^{+}$intercalation in the cathode materials. The reactions during the charge/discharge cycles could be described by the following chemical equations:

$\mathrm{Mn}_{3} \mathrm{O}_{4} \rightarrow 2 \mathrm{MnO}_{2} \cdot x \mathrm{H}_{2} \mathrm{O}+\mathrm{Mn}^{2+}+2 e^{-}$(birnessite)

$\mathrm{MnO}_{2} \cdot x \mathrm{H}_{2} \mathrm{O}+y \mathrm{Zn}^{2+}+2 y e^{-} \leftrightarrow \mathrm{Zn}_{y} \mathrm{MnO}_{2} \cdot x \mathrm{H}_{2} \mathrm{O}$ (Zn-birnessite)

$\mathrm{MnO}_{2} \cdot x \mathrm{H}_{2} \mathrm{O}+\mathrm{H}^{+}+e^{-} \leftrightarrow \mathrm{MnO}(\mathrm{OH})+x \mathrm{H}_{2} \mathrm{O}$

$4 \mathrm{Zn}^{2+}+6 \mathrm{OH}^{-}+\mathrm{SO}_{4}^{2-}+5 \mathrm{H}_{2} \mathrm{O} \leftrightarrow 3 \mathrm{Zn}(\mathrm{OH})_{2} \cdot \mathrm{ZnSO}_{4} \cdot 5 \mathrm{H}_{2} \mathrm{O}$ 


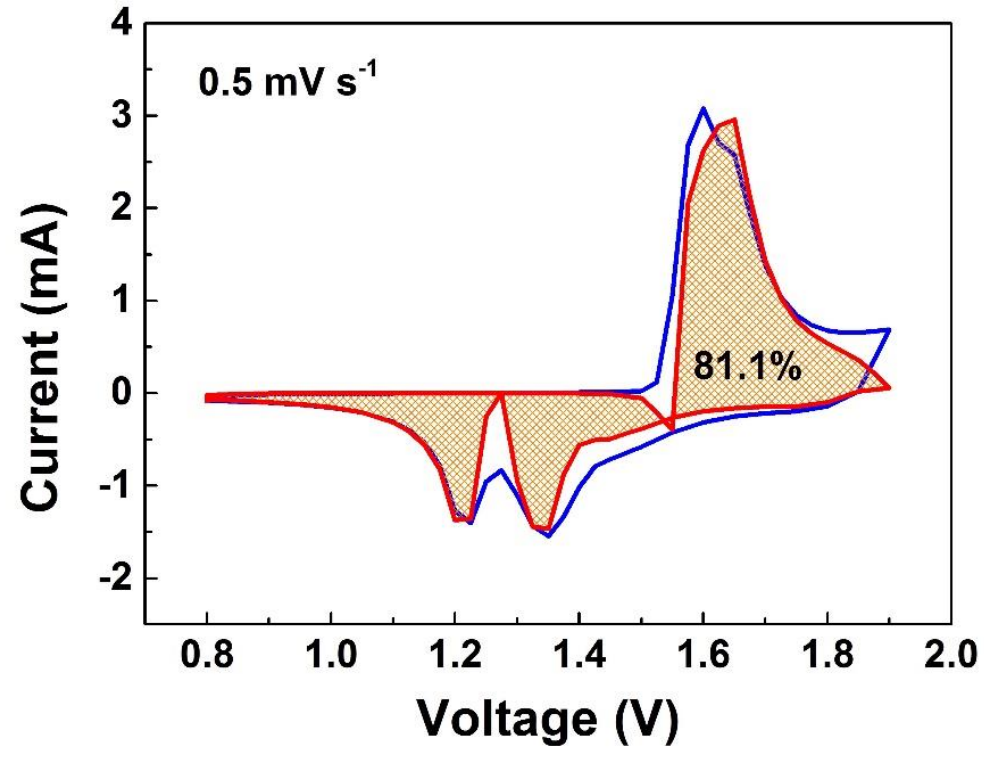

Figure S4. Capacitive contribution (shaded) to the total $\mathrm{Zn}^{2+}$ storage capacity at the scan rate of $0.5 \mathrm{mV} \mathrm{s}^{-1}$ of the $\mathrm{Mn}_{3} \mathrm{O}_{4}$ nanodots. 

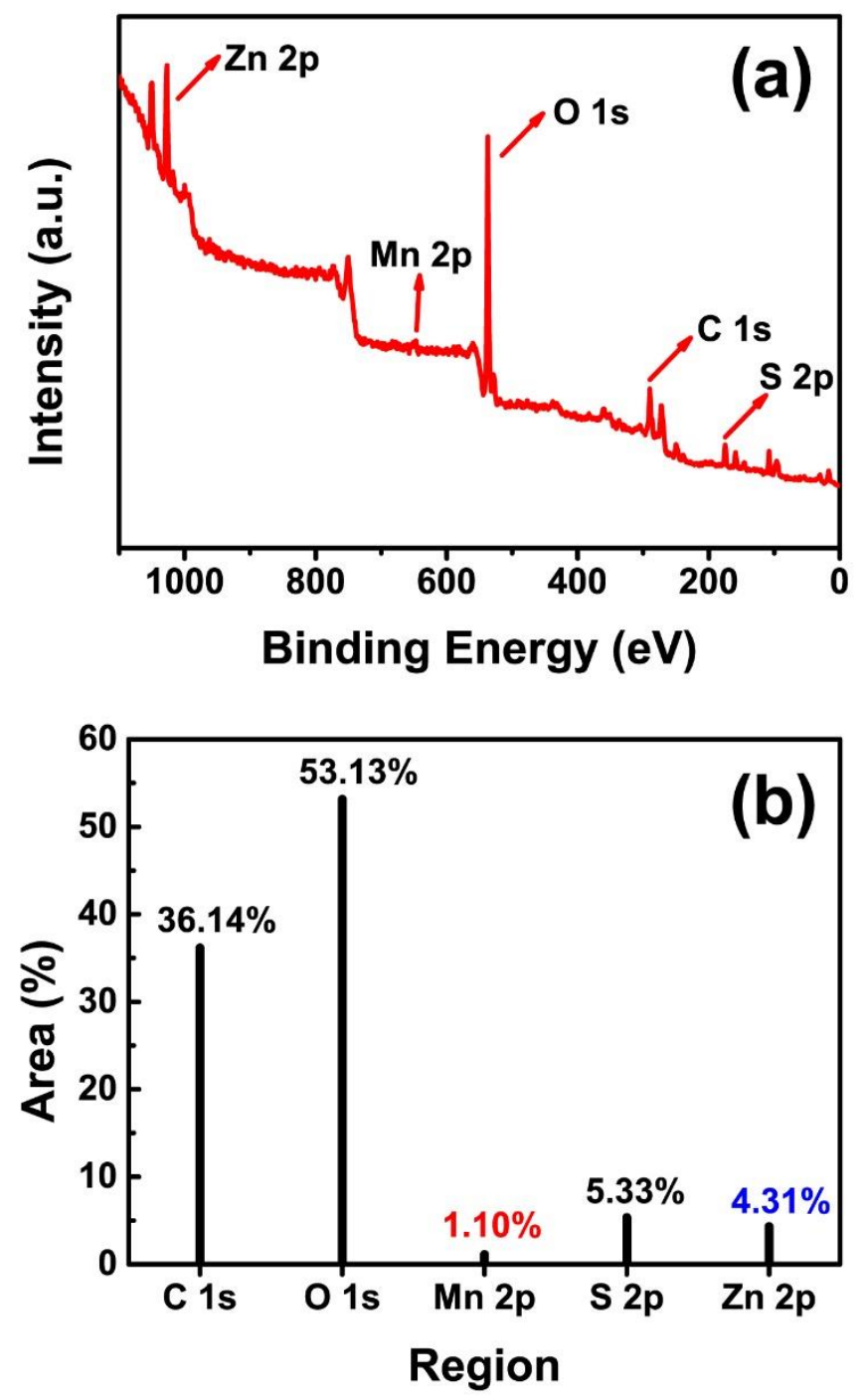

Figure S5. (a) XPS survey spectrum and (b) corresponding peak area ratio of the glass fiber separator disassembled from the Zinc ion secondary battery $\left(2 \mathrm{M} \mathrm{ZnSO} \mathrm{Z}_{4}\right.$ as electrolyte) after 100 cycles at a current density of $0.5 \mathrm{~A} \mathrm{~g}^{-1}$. 


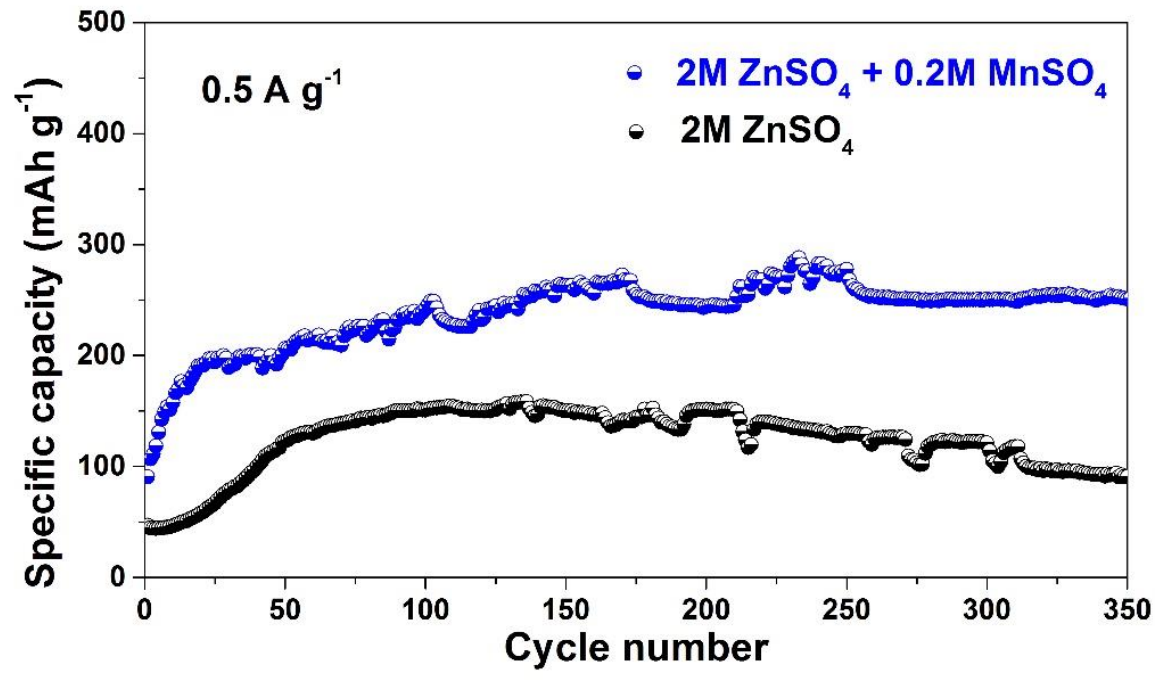

Figure S6. Cycling performance of $\mathrm{Zn}-\mathrm{Mn}_{3} \mathrm{O}_{4}$ nanodots coin cells using $2 \mathrm{M} \mathrm{ZnSO}_{4}+$ $0.2 \mathrm{M} \mathrm{MnSO}_{4}$ and $2 \mathrm{M} \mathrm{ZnSO}_{4}$ as electrolyte at $0.5 \mathrm{~A} \mathrm{~g}^{-1}$, respectively. The $\mathrm{Zn}-\mathrm{Mn}_{3} \mathrm{O}_{4}$ nanodots battery utilizing $2 \mathrm{M} \mathrm{ZnSO}_{4}$ electrolyte shows lower capacity than that of $\mathrm{Zn} / /$ $\mathrm{ZnSO}_{4}+\mathrm{MnSO}_{4} / \mathrm{Mn}_{3} \mathrm{O}_{4}$ nanodots battery. Obvious capacity degradation has also been observed in the $\mathrm{Zn}-\mathrm{Mn}_{3} \mathrm{O}_{4}$ nanodots battery using $2 \mathrm{M} \mathrm{ZnSO}_{4}$ electrolyte after 350 cycles at $0.5 \mathrm{~A} \mathrm{~g}^{-1}$. 


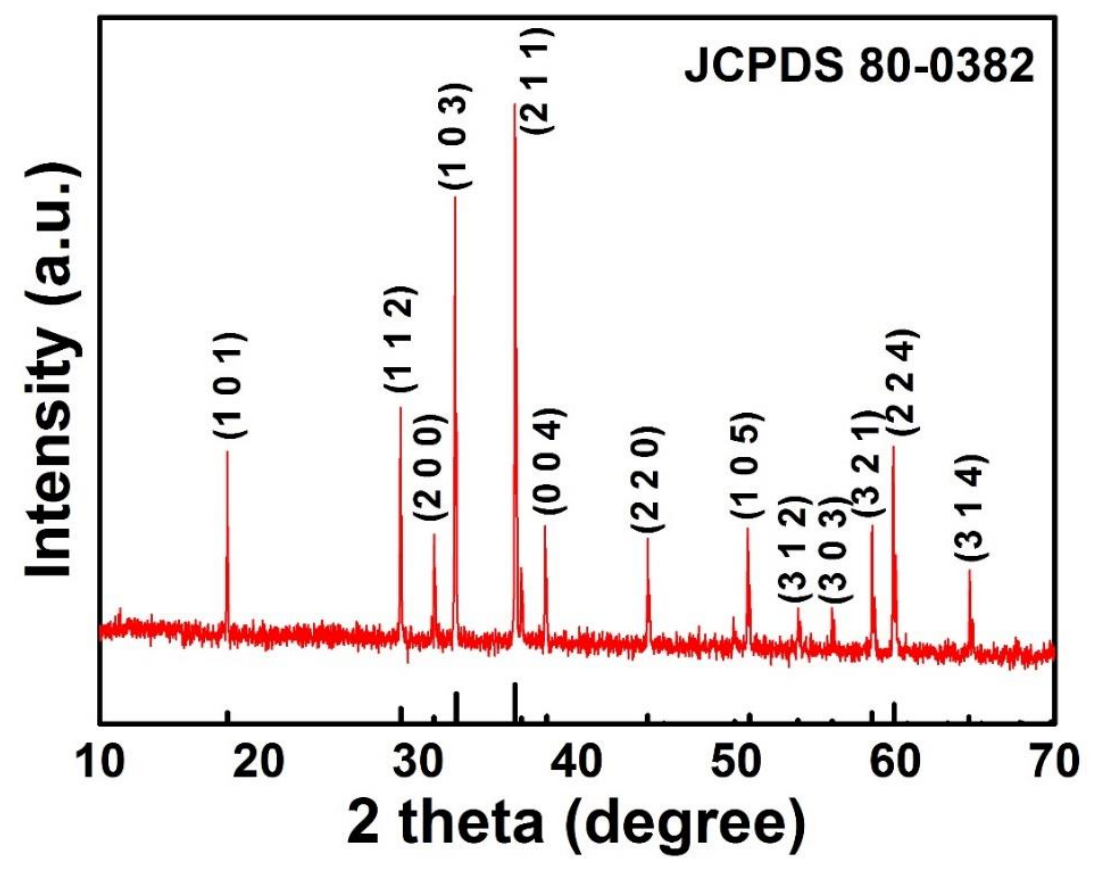

Figure S7. Typical XRD pattern of commercial $\mathrm{Mn}_{3} \mathrm{O}_{4}$ powder (Sinopharm Chemical Reagent Co., Ltd). 


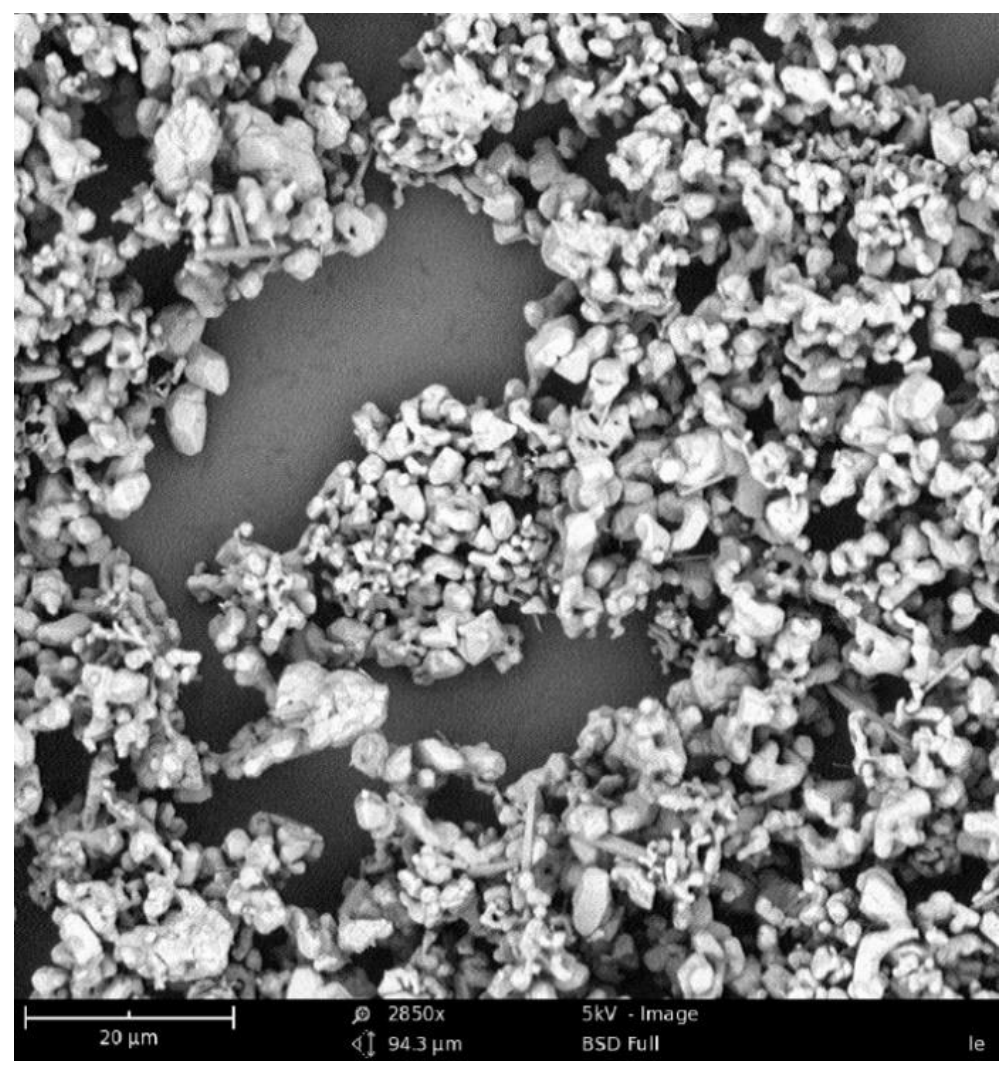

Figure S8. SEM image of commercial $\mathrm{Mn}_{3} \mathrm{O}_{4}$ powder with an average size of $3.3 \mu \mathrm{m}$. 


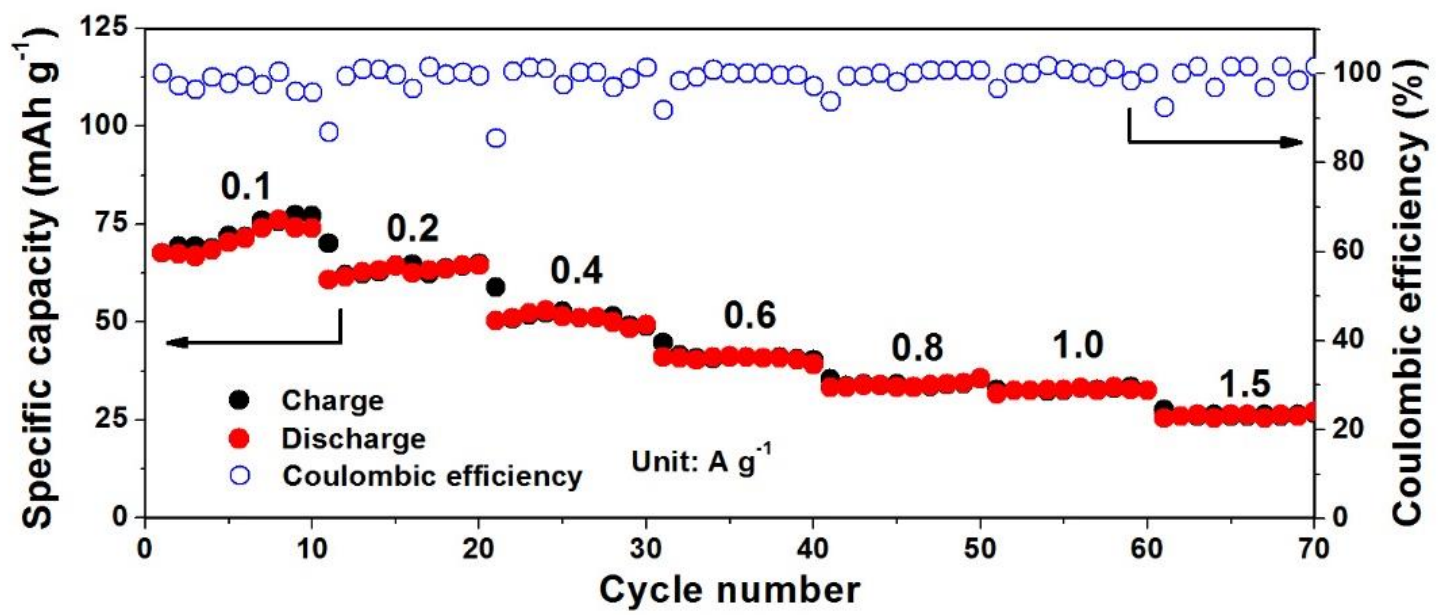

Figure S9. Rate-performance of the $\mathrm{Zn}$ ion battery constructed from commercial $\mathrm{Mn}_{3} \mathrm{O}_{4}$ cathode. 


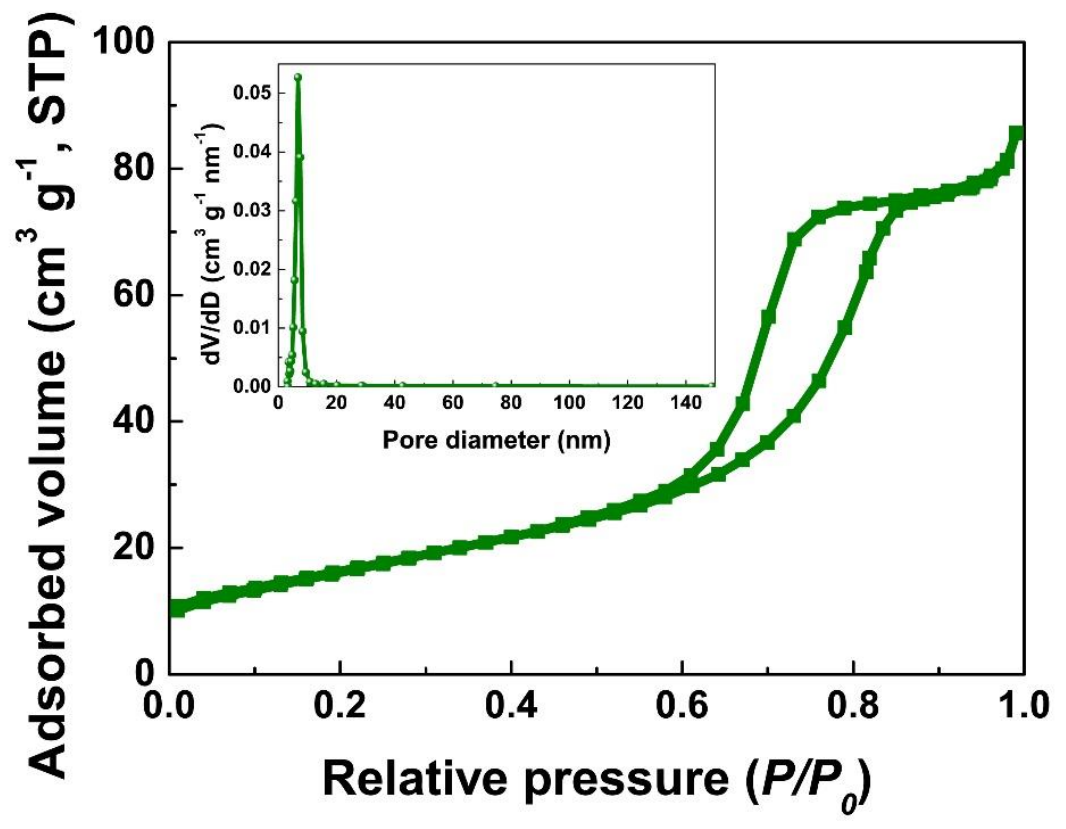

Figure S10. $\mathrm{N}_{2}$ isothermal adsorption curve and corresponding pore size distribution curve (inset) of $\mathrm{Mn}_{3} \mathrm{O}_{4}$ nanodots. 


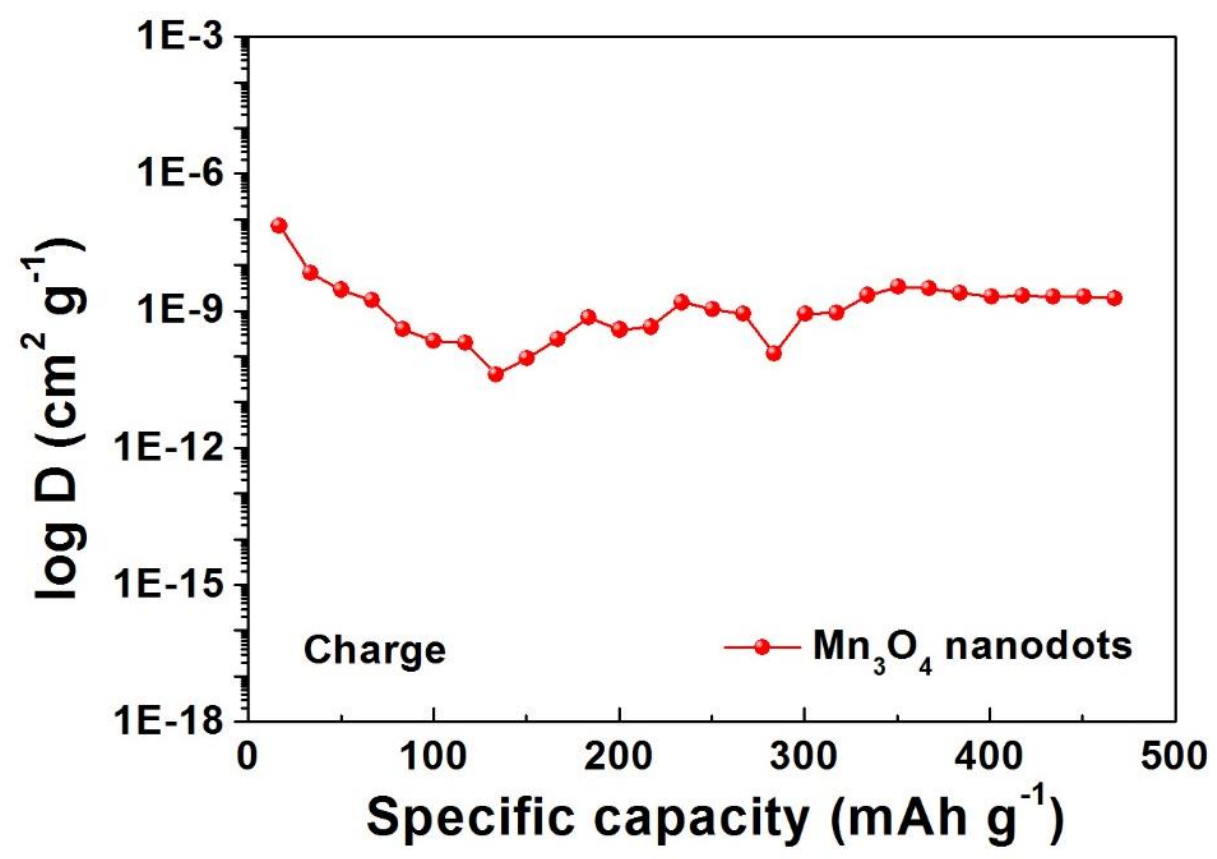

Figure S11. Diffusion coefficient $(D)$ in the charge process calculated by GITT profiles of the $\mathrm{Zn}-\mathrm{Mn}_{3} \mathrm{O}_{4}$ nanodots battery. The average $D_{H}$ and $D_{\mathrm{Zn}}$ value at discharge plateau were calculated to be $6.5 \times 10^{-9} \mathrm{~cm}^{2} \mathrm{~s}^{-1}$ and $2.4 \times 10^{-10} \mathrm{~cm}^{2} \mathrm{~s}^{-1}$ (Figure 5b, 5c), respectively. The obvious turning point at around $1.3 \mathrm{~V}$ (Figure 2d) gradually disappears with the increasing of the current density and could be ascribed to the sudden slowing down of the ion diffusion coefficient. ${ }^{3}$ 


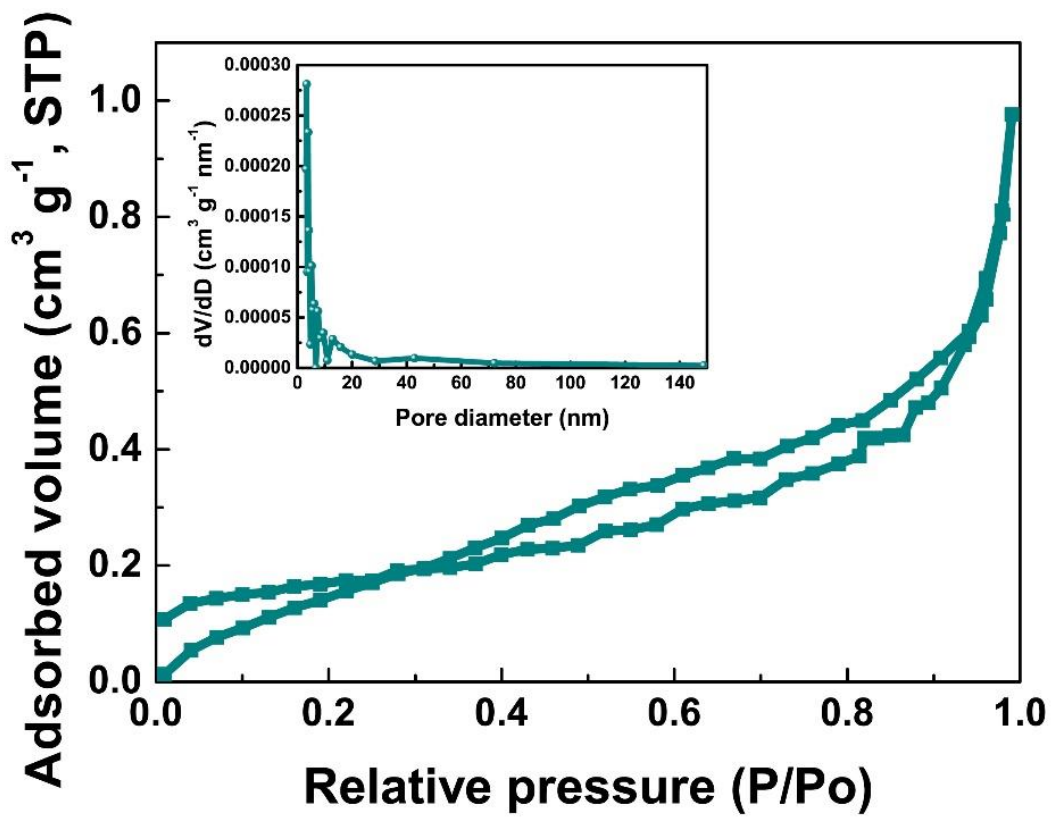

Figure S12. $\mathrm{N}_{2}$ isothermal adsorption curve and corresponding pore size distribution curve (inset) of commercial $\mathrm{Mn}_{3} \mathrm{O}_{4}$ powder. 

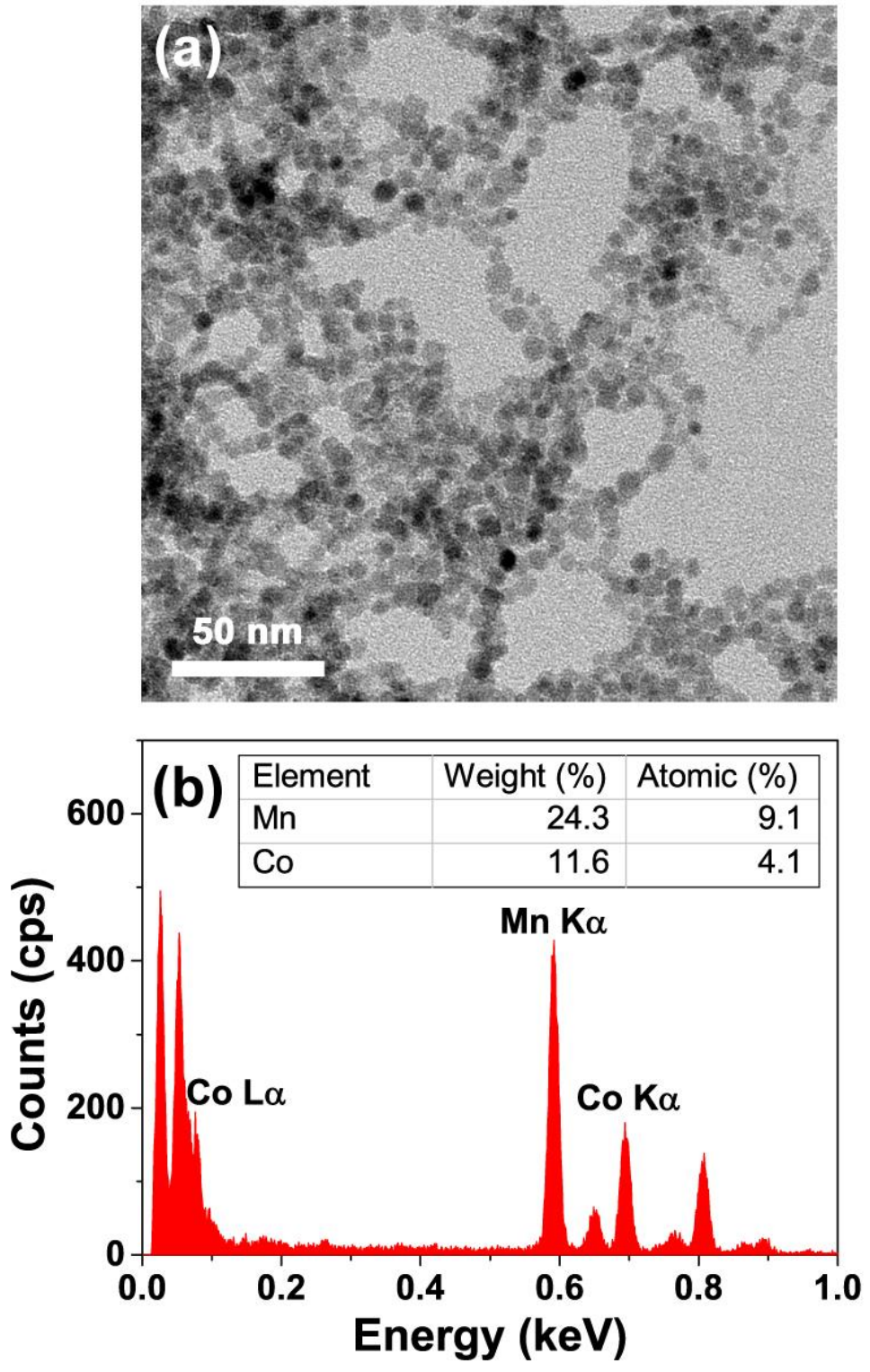

Figure S13. (a) Characteristic TEM image and (b) EDS spectra of $\mathrm{CoMn}_{2} \mathrm{O}_{4}$ nanodots. 

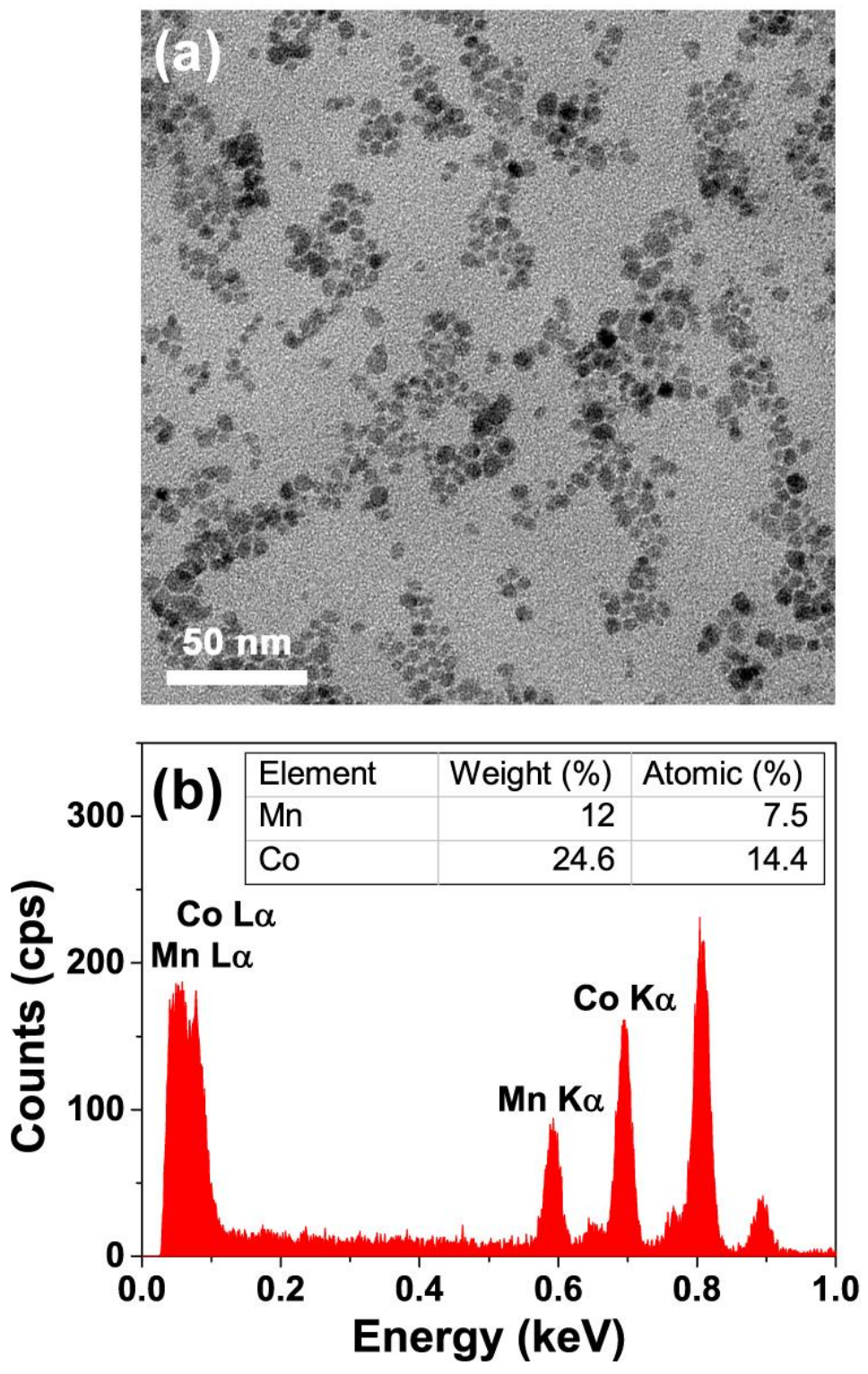

Figure S14. (a) Characteristic TEM image and (b) EDS spectra of $\mathrm{MnCo}_{2} \mathrm{O}_{4}$ nanodots. 

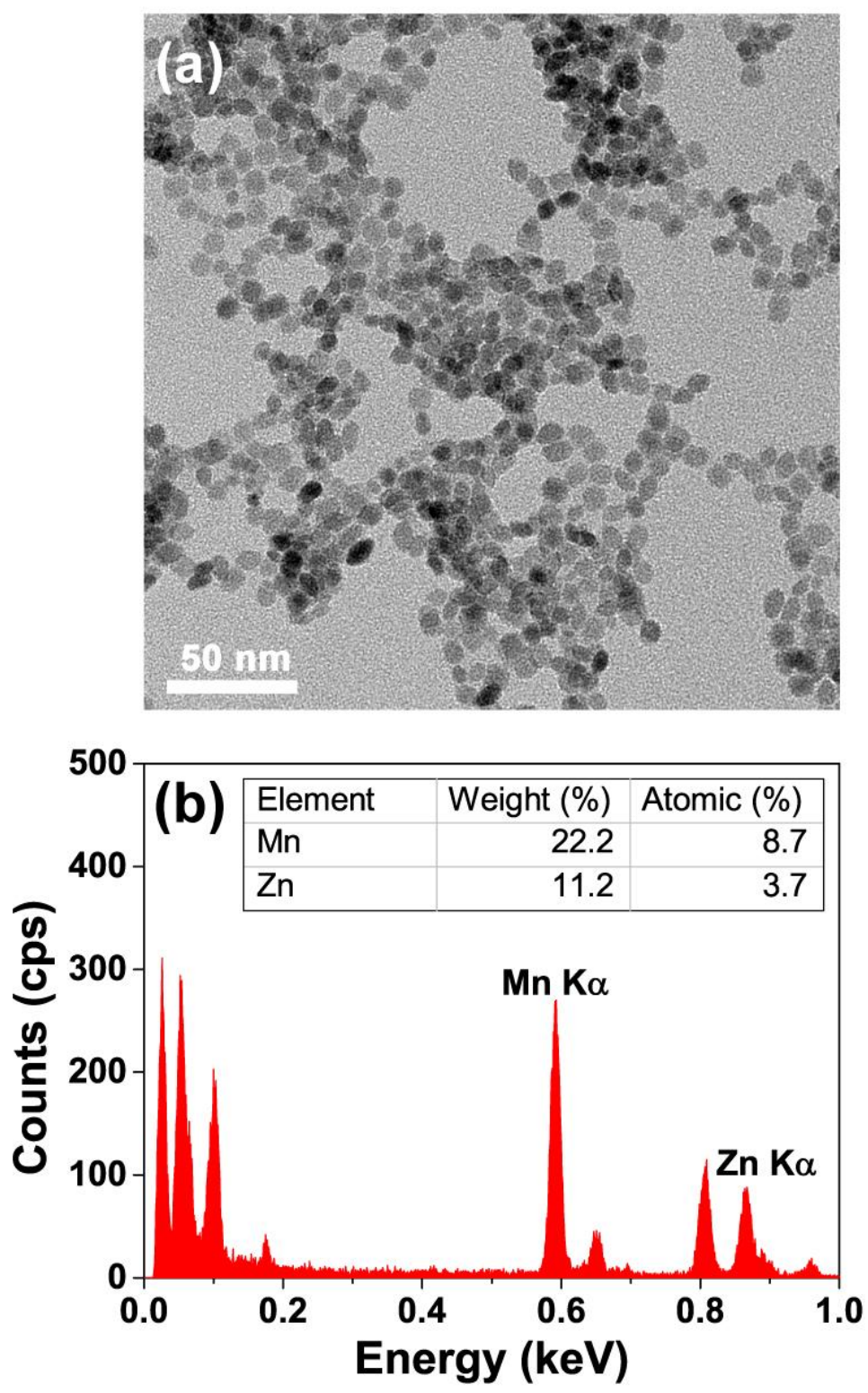

Figure S15. (a) Characteristic TEM image and (b) EDS spectra of $\mathrm{ZnMn}_{2} \mathrm{O}_{4}$ nanodots. 


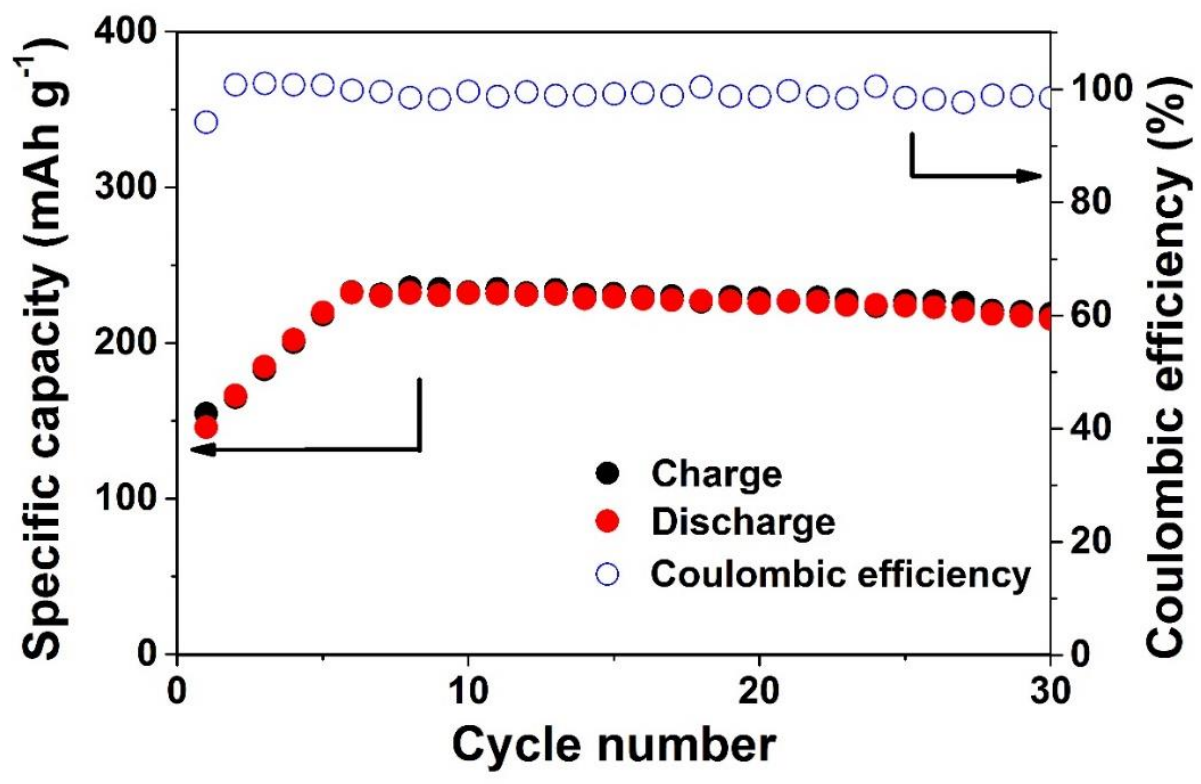

Figure S16. Cycling performance of $\mathrm{CoMn}_{2} \mathrm{O}_{4}$ nanodots at a current density of $0.1 \mathrm{~A} \mathrm{~g}^{-1}$. Note that the capacity fading is fast after 30 cycles due to the poor activity of cobalt (II) in mild aqueous electrolyte conditions. 


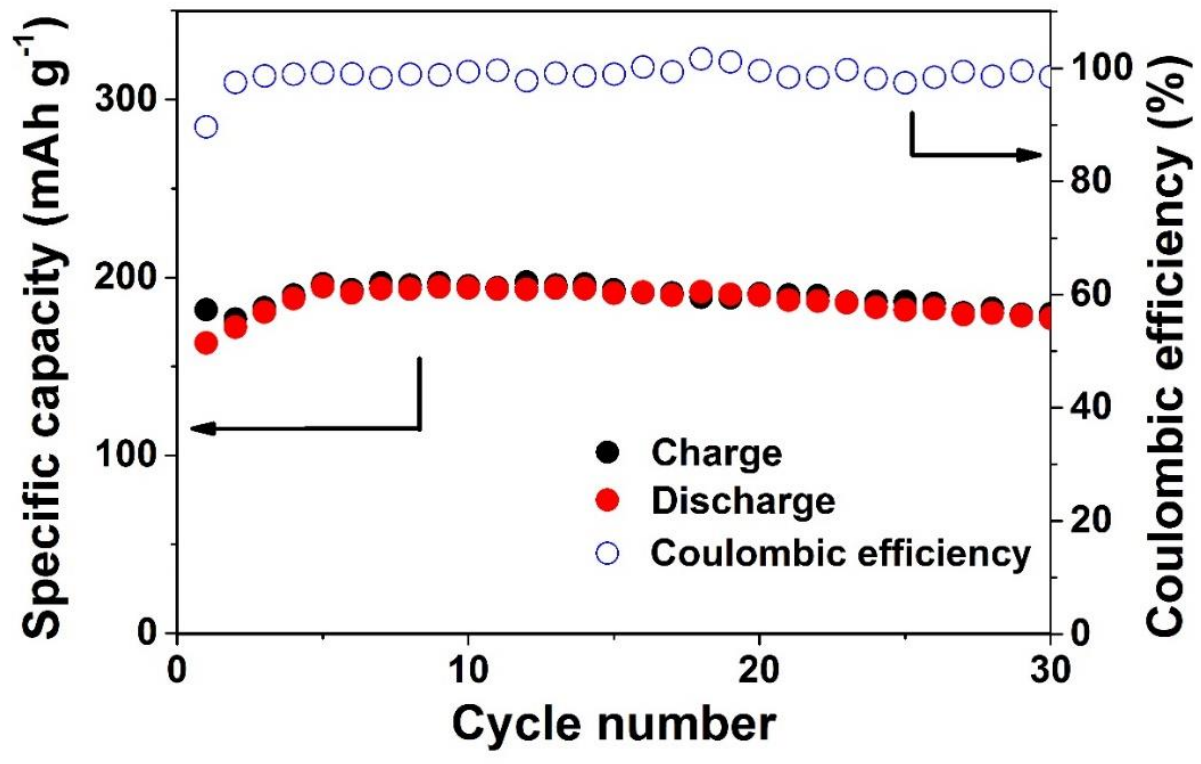

Figure S17. Cycling performance of $\mathrm{MnCo}_{2} \mathrm{O}_{4}$ nanodots at a current density of $0.1 \mathrm{~A} \mathrm{~g}^{-1}$. Note that the capacity fading is fast after 30 cycles due to the poor activity of cobalt (II) in mild aqueous electrolyte conditions. 


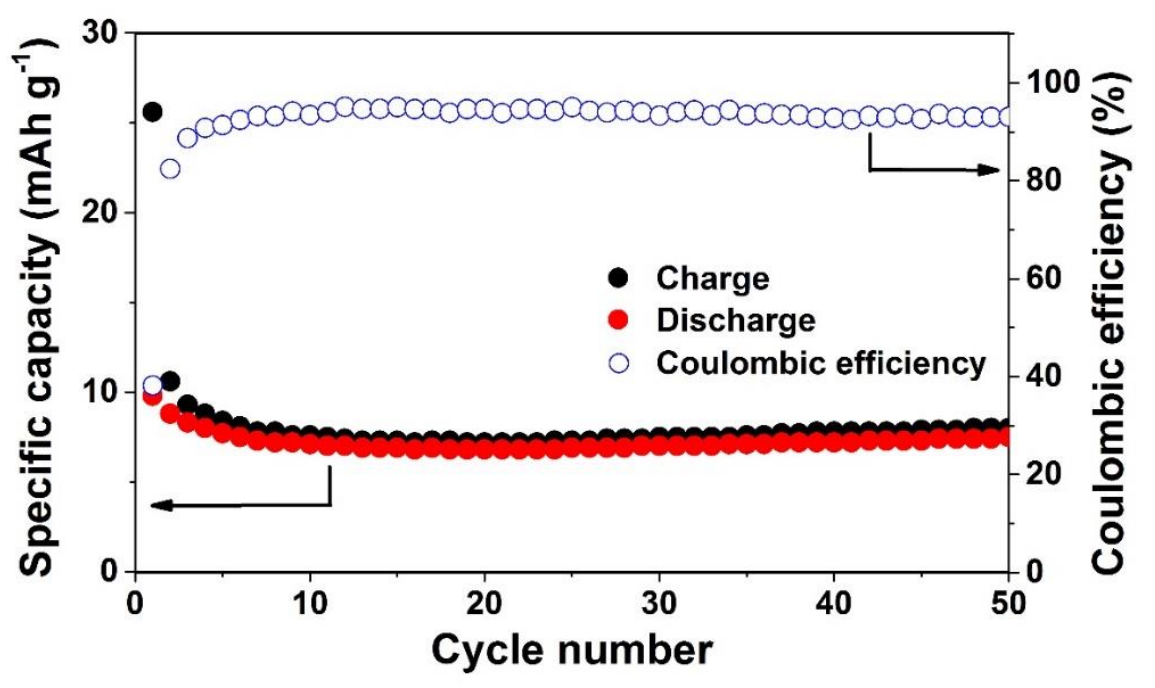

Figure S18. Cycling performance of $\mathrm{Co}_{3} \mathrm{O}_{4}$ nanodots at a current density of $0.1 \mathrm{~A} \mathrm{~g}^{-1}$. 


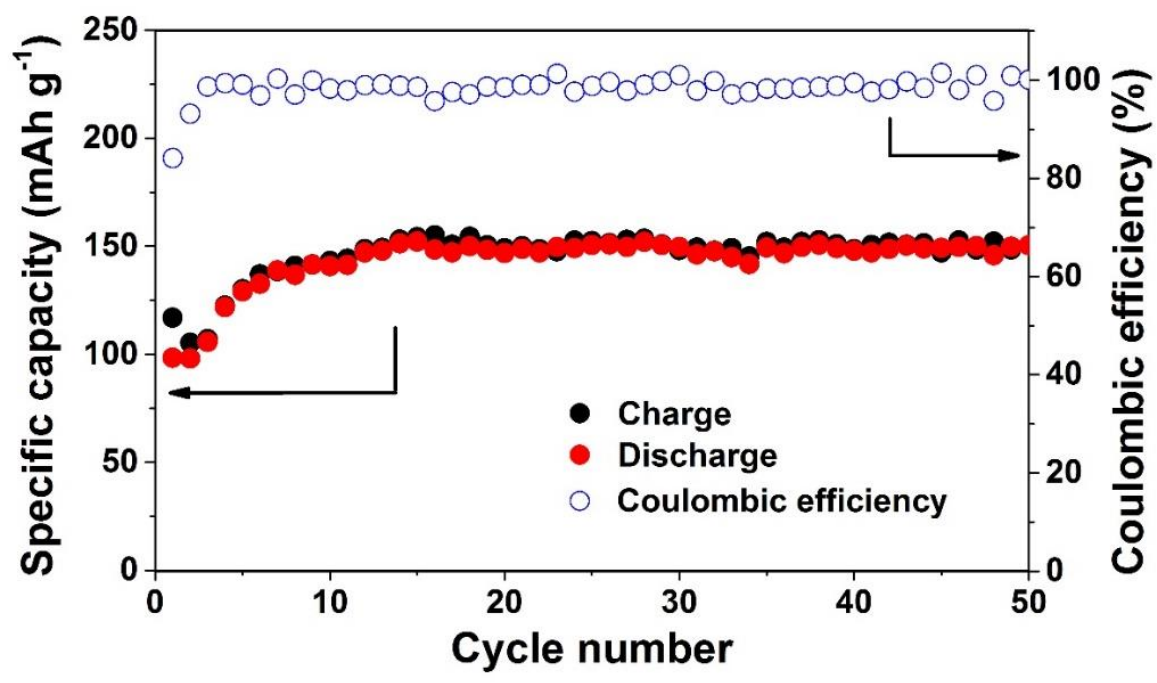

Figure S19. Cycling performance of $\mathrm{ZnMn}_{2} \mathrm{O}_{4}$ nanodots at a current density of $0.1 \mathrm{~A} \mathrm{~g}^{-1}$. 
Table S1. The comparison for rate performance of cathode materials in ZIBs.

\begin{tabular}{|c|c|c|c|}
\hline Cathode material & Electrolyte & Rate performance & Ref\# \\
\hline $\mathrm{Mn}_{3} \mathrm{O}_{4}$ nanodots & $\begin{array}{l}2 \mathrm{M} \mathrm{ZnSO}_{4} \\
+0.2 \mathrm{M} \mathrm{MnSO}_{4}\end{array}$ & $\begin{array}{l}386.7 \mathrm{~mA} \mathrm{~h} \mathrm{~g}^{-1} \text { at } 0.1 \mathrm{~A} \mathrm{~g}^{-1} \\
336.2 \mathrm{~mA} \mathrm{~h} \mathrm{~g}^{-1} \text { at } 0.2 \mathrm{~A} \mathrm{~g}^{-1} \\
284.4 \mathrm{~mA} \mathrm{~h} \mathrm{~g} \text { at } 0.4 \mathrm{~A} \mathrm{~g}^{-1} \\
245.7 \mathrm{~mA} \mathrm{~h} \mathrm{~g}^{-1} \text { at } 0.6 \mathrm{~A} \mathrm{~g}^{-1}\end{array}$ & This work. \\
\hline $\mathrm{Mn}_{3} \mathrm{O}_{4}(50 \sim 100 \mathrm{~nm})$ & $2 \mathrm{M} \mathrm{ZnSO}_{4}$ & $\begin{array}{l}232.9 \mathrm{~mA} \mathrm{~h} \mathrm{~g}^{-1} \text { at } 0.1 \mathrm{~A} \mathrm{~g}^{-1} \\
232 \mathrm{~mA} \mathrm{~h} \mathrm{~g} \text { at } 0.2 \mathrm{~A} \mathrm{~g}^{-1} \\
195 \mathrm{~mA} \mathrm{~h} \mathrm{~g} \text { at } 0.5 \mathrm{~A} \mathrm{~g}^{-1}\end{array}$ & 2 \\
\hline $\mathrm{Mn}_{3} \mathrm{O}_{4}$ nanoflower & $\begin{array}{l}2 \mathrm{M} \mathrm{ZnSO}_{4} \\
+0.1 \mathrm{M} \mathrm{MnSO}_{4}\end{array}$ & $\begin{array}{l}296 \mathrm{~mA} \mathrm{~h} \mathrm{~g}^{-1} \text { at } 0.1 \mathrm{~A} \mathrm{~g}^{-1} \\
185 \mathrm{~mA} \mathrm{~h} \mathrm{~g} \text { at } 0.3 \mathrm{~A} \mathrm{~g}^{-1} \\
125 \mathrm{~mA} \mathrm{~h} \mathrm{~g} \text { at } 0.5 \mathrm{~A} \mathrm{~g}^{-1}\end{array}$ & 4 \\
\hline $\mathrm{Mn}_{3} \mathrm{O}_{4} @ \mathrm{CNW}$ & $\begin{array}{l}1 \mathrm{M} \mathrm{ZnSO}_{4} \\
+0.1 \mathrm{M} \mathrm{MnSO}_{4} \\
+0.1 \mathrm{M} \mathrm{NaSO}_{4}\end{array}$ & $\begin{array}{l}237 \mathrm{~mA} \mathrm{~h} \mathrm{~g}^{-1} \text { at } 0.12 \mathrm{~A} \mathrm{~g}^{-1} \\
180 \mathrm{~mA} \mathrm{~h} \mathrm{~g} \text { at } 0.24 \mathrm{~A} \mathrm{~g}^{-1} \\
155 \mathrm{~mA} \mathrm{~h} \mathrm{~g} \text { at } 0.48 \mathrm{~A} \mathrm{~g}^{-1}\end{array}$ & 5 \\
\hline $\mathrm{Mn}_{3} \mathrm{O}_{4}(150 \mathrm{~nm})$ & $\begin{array}{l}1 \mathrm{M} \mathrm{ZnSO}_{4} \\
+0.1 \mathrm{M} \mathrm{MnSO}_{4}\end{array}$ & $\begin{array}{l}221 \mathrm{~mA} \mathrm{~h} \mathrm{~g}^{-1} \text { at } 0.1 \mathrm{~A} \mathrm{~g}^{-1} \\
182 \mathrm{~mA} \mathrm{~h} \mathrm{~g}^{-1} \text { at } 0.2 \mathrm{~A} \mathrm{~g}^{-1} \\
132 \mathrm{~mA} \mathrm{~h} \mathrm{~g} \text { at } 0.5 \mathrm{~A} \mathrm{~g}^{-1}\end{array}$ & 6 \\
\hline Commercial $\mathrm{Mn}_{3} \mathrm{O}_{4}$ & $\begin{array}{l}2 \mathrm{M} \mathrm{ZnSO}_{4} \\
+0.2 \mathrm{M} \mathrm{MnSO}_{4}\end{array}$ & $\begin{array}{l}74.1 \mathrm{~mA} \mathrm{~h} \mathrm{~g}^{-1} \text { at } 0.1 \mathrm{~A} \mathrm{~g}^{-1} \\
64.4 \mathrm{~mA} \mathrm{~h} \mathrm{~g} \text { at } 0.2 \mathrm{~A} \mathrm{~g}^{-1} \\
52.9 \mathrm{~mA} \mathrm{~h} \mathrm{~g} \text { at } 0.4 \mathrm{~A} \mathrm{~g}^{-1} \\
40.8 \mathrm{~mA} \mathrm{~h} \mathrm{~g} \text { at } 0.6 \mathrm{~A} \mathrm{~g}^{-1}\end{array}$ & I \\
\hline
\end{tabular}


Table S2. The crystallographic information of the as-prepared spinel oxide nanodots.

\begin{tabular}{|c|c|c|}
\hline Composition & Lattice parameters & Crystal system \\
\hline $\mathrm{Mn}_{3} \mathrm{O}_{4}$ & $a=b=5.765 \AA, \mathrm{c}=9.442 \AA, \alpha=\beta=\gamma=90^{\circ}$ & tetragonal \\
\hline $\mathrm{CoMn}_{2} \mathrm{O}_{4}$ & $a=b=8.09 \stackrel{\mathrm{A}}{ }, \mathrm{c}=9.27 \stackrel{\mathrm{A}}{ }, \alpha=\beta=\gamma=90^{\circ}$ & tetragonal \\
\hline $\mathrm{MnCo}_{2} \mathrm{O}_{4.5}$ & $a=b=\mathrm{c}=8.08 \stackrel{\mathrm{A}}{ }, \alpha=\beta=\gamma=90^{\circ}$ & cubic \\
\hline $\mathrm{Co}_{3} \mathrm{O}_{4}$ & $a=b=\mathrm{c}=8.084 \AA, \alpha=\beta=\gamma=90^{\circ}$ & cubic \\
\hline $\mathrm{ZnMn}_{2} \mathrm{O}_{4}$ & $a=b=5.7204 \AA, \mathrm{c}=9.245 \AA, \alpha=\beta=\gamma=90^{\circ}$ & tetragonal \\
\hline
\end{tabular}

\section{REFERENCES}

(1) Huang, J.; Wang, Z.; Hou, M.; Dong, X.; Liu, Y.; Wang, Y.; Xia, Y. PolyanilineIntercalated Manganese Dioxide Nanolayers as a High-Performance Cathode Material for an Aqueous Zinc-ion Battery. Nat. Commun. 2018, 9, 2906.

(2) Hao, J.; Mou, J.; Zhang, J.; Dong, L.; Liu, W.; Xu, C.; Kang, F. Electrochemically Induced Spinel-Layered Phase Transition of $\mathrm{Mn}_{3} \mathrm{O}_{4}$ in High Performance Neutral Aqueous Rechargeable Zinc Battery. Electrochim. Acta 2018, 259, 170.

(3) Liu, Y.; Zhou, X.; Liu, R.; Li, X.; Bai, Y.; Xiao, H.; Wang, Y.; Yuan, G. Tailoring Three-Dimensional Composite Architecture for Advanced Zinc-Ion Batteries. ACS Appl. Mater. Interfaces 2019, 11, 19191-19199.

(4) Zhu, C.; Fang, G.; Zhou, J.; Guo, J.; Wang, Z.; Wang, C.; Li, J.; Tang, Y.; Liang, S. Binder-Free Stainless Steel@ $\mathrm{Mn}_{3} \mathrm{O}_{4}$ Nanoflower Composite: a High-Activity Aqueous Zinc-Ion Battery Cathode with High-Capacity and Long-Cycle-Life. J. Mater. Chem. A 2018, 6, 9677.

(5) Ma, L.; Li, L.; Liu, Y.; Zhu, J.; Meng, T.; Zhang, H.; Jiang, J.; Li, C. M. Building Better Rechargeable Zn-Mn Batteries with a Highly Active $\mathrm{Mn}_{3} \mathrm{O}_{4} /$ Carbon Nanowire Cathode and Neutral $\mathrm{Na}_{2} \mathrm{SO}_{4} / \mathrm{MnSO}_{4}$ Electrolyte. Chem. Commun. 2018, 54, 10835.

(6) Wang, L.; Cao, X.; Xu, L.; Chen, J.; Zheng, J. Transformed Akhtenskite $\mathrm{MnO}_{2}$ from $\mathrm{Mn}_{3} \mathrm{O}_{4}$ as Cathode for a Rechargeable Aqueous Zinc Ion Battery. ACS Sustainable Chem. Eng. 2018, 6, 16055. 